\title{
S3-Leitlinie zur Diagnostik, Beratung und Behandlung im Kontext von Geschlechtsinkongruenz, Geschlechtsdysphorie und Trans-Gesundheit
}

Hintergrund, Methode und zentrale Empfehlungen

\section{S3-Guideline on Diagnosis, Counselling and Treatment in the Context of Gender Incongruence, Gender Dysphoria and Trans Health}

\author{
Background, Methods and Key Recommendations
}

\author{
Autor_innen \\ Timo O. Nieder ${ }^{1}$ und Bernhard Strauß ${ }^{2}$ \\ Institute \\ 1 Institut für Sexualforschung und Forensische Psychiatrie, \\ Universitätsklinikum Hamburg-Eppendorf \\ 2 Institut für Psychosoziale Medizin und Psychotherapie, \\ Klinikum der Friedrich-Schiller-Universität Jena \\ Schlüsselwörter \\ Gesundheitsversorgung; Konsens; Leitlinien; Transgender; \\ Transsexualität \\ Keywords \\ consensus; guidelines; health care; transgender; \\ transsexualism \\ Bibliografie \\ DOI https://doi.org/10.1055/a-0895-8176 \\ Z Sexualforsch 2019; 32: 70-79 \\ (c) Georg Thieme Verlag KG Stuttgart · New York \\ ISSN 0932-8114 \\ Korrespondenzadresse \\ Dr. phil. Timo O. Nieder \\ Universitätsklinikum Hamburg-Eppendorf \\ Institut für Sexualforschung und Forensische Psychiatrie \\ Martinistraße 52 \\ 20246 Hamburg \\ tnieder@uke.de
}

\section{ZUSAMMENFASSUNG}

Einleitung Am 9. Oktober 2018 wurde die Leitlinie „Geschlechtsinkongruenz, Geschlechtsdysphorie und TransGesundheit: Diagnostik, Beratung und Behandlung " nach methodischer Prüfung von der Arbeitsgemeinschaft der Wissenschaftlichen Medizinischen Fachgesellschaften (AWMF) mit S3-Status in das Leitlinienregister aufgenommen und online veröffentlicht.
Forschungsziele Die Leitlinie verfolgt das Ziel, die „Standards zur Begutachtung und Behandlung von Transsexuellen“ aus dem Jahr 1997 an die gegenwärtigen Bedingungen und Forschungsergebnisse anzupassen und sie für eine adäquate Versorgung in Deutschland nutzbar zu machen.

Methoden Die Behandlungsempfehlungen der Leitlinie basieren auf empirischer Evidenz, die systematisch recherchiert und bewertet wurde. Die für den Kreis der Adressat_innen repräsentative Leitliniengruppe hat gemeinsam mit einer Interessensvertretung von trans Menschen im Zuge einer strukturierten Konsensfindung 100 Empfehlungen konsentiert.

Ergebnisse Die Leitlinie soll das Feld der Trans-Gesundheitsversorgung individualisieren und damit auch deregulieren. Optionen für eine zielführende Behandlung der Geschlechtsinkongruenz und/oder Geschlechtsdysphorie werden aufgezeigt. Anschließend wird ein empirisch und klinisch fundiertes Vorgehen empfohlen, das auf individuelle Bedingungen bei der Behandlung bedarfsgerecht eingehen kann.

Schlussfolgerung Die Leitlinie reflektiert aktuelle internationale Standards der Trans-Gesundheitsversorgung auf Basis empirischer Evidenz und bezieht sie auf das deutsche Gesundheitssystem. Ihre Anwendung sollte mit klinisch-therapeutischer Expertise abgesichert werden.

\section{ABSTRACT}

Introduction On October 9, 2018, the German guidelines "Gender Incongruence, Gender Dysphoria and Trans Health: Diagnostics, Counselling and Treatment" were published online after a methodological review by the Association of Scientific Medical Societies (AWMF) with S3 status.

Objectives The objective of the guideline group was to adapt the 1997 "Standards for Treatment and Expert Opinion on Transsexuals" to current scientific developments and research results and to make them useful for the provision of appropriate health care in Germany. 
Methods The treatment recommendations of the guideline are based on empirical evidence which was systematically researched and evaluated. In a structured consensus process, the guideline group, who is representative for the target group, as well as a stakeholder group of trans people in Germany agreed on 100 recommendations.

Results The guideline aims to individualize and deregulate the field of trans health care. Reasonable options for the treatment of gender incongruence and/or gender dysphoria are outlined. A procedure that is based on the empirical and clinical evidence is then recommended which can be adapted to individual conditions in the course of treatment.

Conclusion The guideline reflects the current international state of trans health care on the basis of empirical evidence and puts it in relation to the German health care system. Its application should be backed up by clinical and therapeutic expertise.
Am 9. Oktober 2018 wurde die Leitlinie „Geschlechtsinkongruenz, Geschlechtsdysphorie und Trans-Gesundheit: Diagnostik, Beratung und Behandlung “ nach methodischer Prüfung von der Arbeitsgemeinschaft der Wissenschaftlichen Medizinischen Fachgesellschaften (AWMF) mit S3-Status in das Leitlinienregister aufgenommen und online veröffentlicht. Die Veröffentlichung der Leitlinie markierte den Endpunkt einer nach Vorgaben der AWMF durchgeführten Leitlinienentwicklung, die im Jahr 2012 auf Initiative der Deutschen Gesellschaft für Sexualforschung (DGfS) begonnen hatte.

\section{Situation in Deutschland}

Bis zum Zeitpunkt der Veröffentlichung der Leitlinie waren die Standards der Behandlung und Begutachtung von Transsexuellen der Deutschen Gesellschaft für Sexualforschung, der Akademie für Sexualmedizin und der Gesellschaft für Sexualwissenschaft von Becker et al. (1997) der Maßstab für Behandlungsentscheidungen, wenngleich diese schon länger als überarbeitungsbedürftig markiert wurden (Nieder und Strauß 2014: 62). Daneben hat die Begutachtungsrichtlinie des Medizinischen Dienstes des Spitzenverbandes Bund der Krankenkassen (MDS 2009) einen prägenden Effekt auf die Behandlung: Sie schreibt die Voraussetzungen für die Kostenübernahme fest, die für alle Behandlungssuchenden gleichermaßen gelten sollen (Nieder et al. 2014b) und führt zu einer Vielzahl von Problemen für Behandlungssuchende und Behandelnde gleichermaßen (Hamm und Sauer 2014). Die Krankenkassen treffen ihre Entscheidungen zur Kostenübernahme der beantragten Leistungen in der Regel in Abhängigkeit von einer einzelfallbezogenen sozialmedizinischen Begutachtung, für deren Erstellung die MDS-Begutachtungsrichtlinie (MDS 2009) verbindlich ist. Zu den Voraussetzungen für die Kostenübernahme gehören eine Phase der Diagnostik und Behandlungsplanung, die Durchführung einer Psychotherapie von mindestens zwölf, eher 18 Monaten Dauer (einhergehend mit dem Erreichen der für alle Behandlungssuchenden pauschal geltenden Psychotherapieziele) sowie die Alltagserprobung im Sinne der „Lebbarkeit der gewünschten Geschlechtsrolle“ (MDS 2009: 10). Vor allen somatischen Behandlungen muss „in jedem Fall eine psychiatrische/psychotherapeutische Behandlung “ erfolgen (MDS 2009: 12). Zudem fordert die MDS-Begutachtungsrichtlinie einen „Alltagstest [von] in der Regel mindestens 12 Monate[n]“ Dauer: Vor Beginn einer Behandlung mit Sexualhormonen soll die betreffende Person „das Leben in der gewünschten Geschlechtsrolle erprobt“ haben (MDS 2009: 18). Insgesamt reguliert auf diese Weise eine Vielzahl zu erfüllender Voraussetzungen die Kostenübernahme für indizierte Behandlungen, ohne dass die Notwendigkeit der Voraussetzung im Sinne ihrer Wirksamkeit (z. B. Reduktion des spe- zifischen Leidens) empirisch fundiert ist. Vielmehr häufen sich die Belege von systematischer Diskriminierung und Destabilisierung von trans ${ }^{1}$ Personen im und durch das Gesundheitssystem (Hamm und Sauer 2014; Loos et al. 2016).

Die Leitliniengruppe hat mit der S3-Leitlinie das Ziel verfolgt, die "Standards zur Begutachtung und Behandlung von Transsexuellen“ (Becker et al. 1997) zu aktualisieren und für eine zielführende Versorgung in Deutschland nutzbar zu machen. Als zielführend wird dabei eine Versorgung erachtet, mit deren Hilfe es den betreffenden Menschen gelingt, ihr Geschlecht möglichst selbstbestimmt zu leben. Dabei hat sich die Leitliniengruppe, analog zur Ausrichtung der Standards von 1997, einen psychosozialen Schwerpunkt gesetzt, der die Auswahl der beteiligten Fachgesellschaften und Berufsverbände bedingt hat. Zudem ist der psychosoziale Rahmen für das Wohlbefinden und die Lebensqualität sowie für ein erhöhtes Kongruenzerleben der Behandlungssuchenden neben den somatischen Behandlungen von Bedeutung (zuletzt Köhler et al. 2019). Die Behandlungsempfehlungen sollten auf empirischer Evidenz basieren, weshalb systematisch nach interventionsbezogener Primärliteratur gesucht wurde. Beteiligt wurden Fachgesellschaften und Berufsverbände aus dem Bereich der psychosozialen Medizin und eine Interessensvertretung von trans Menschen. Zudem kommentierten Fachgesellschaften aus dem Bereich der somatischen Medizin $^{2}$ die Leitlinie. So sollte ein breiter Konsens erreicht werden. Im Jahr 2023 muss die Leitlinie schließlich aktualisiert werden, um gültig zu bleiben.

1 Analog zur Leitlinie verwendet der vorliegende Text Trans als inklusive Kurzform, wenn die betreffenden Menschen ihre Geschlechtsidentität inkongruent zu ihren körperlichen Geschlechtsmerkmalen erleben, diese aber der Norm der Zweigeschlechtlichkeit entsprechen. Als Kurzform soll sie den verschiedenen Ausprägungen von Geschlechtlichkeit Rechnung tragen können, wohlwissend, dass es nicht die eine trans Community gibt, sondern mehrere Communitys mit verschiedenen Präferenzen für Begriffe. Die Norm der Zweigeschlechtlichkeit bleibt allerdings für alle (zwangsweise) die Bezugsgröße: für Menschen, die sich von ihr abgrenzen (wollen) oder für Menschen, deren körperliche Geschlechtsmerkmale entweder zu ihrer Geschlechtsidentität passen oder von dieser abweichen.

2 Die folgenden Fachgesellschaften wurden vor der endgültigen Konsentierung einbezogen und haben die Leitlinien kommentiert und positiv bewertet: Deutsche Gesellschaft der Plastischen, Rekonstruktiven und Ästhetischen Chirurgen (DGPRÄC), Deutsche Gesellschaft für Andrologie (DGA), Deutsche Gesellschaft für HNO-Heilkunde, Kopf- und Hals-Chirurgie (DGHNO-KHC), Deutsche Gesellschaft für Humangenetik (GfH), Deutsche Gesellschaft für Sozialmedizin und Prävention (DGSMP), Deutsche Gesellschaft für Urologie (DGU). 


\section{Internationale Entwicklungen}

Seit der Veröffentlichung der Behandlungsstandards von Becker et al. (1997) haben sich im Zusammenhang mit der Gesundheitsversorgung für trans Menschen weltweit weitreichende Änderungen ergeben. Hierzu gehörten zunächst die „Standards of Care“ (SoC) der World Professional Association for Transgender Health (WPATH), die 2011 in ihrer siebten Version herausgegeben wurden (Coleman et al. 2012; in deutscher Übersetzung: Richter-Appelt und Nieder 2014). Seit 2018 wird bereits an der achten Version gearbeitet, diesmal von einer Arbeitsgruppe, die internationaler und trans-inklusiver besetzt ist als bei den vorherigen SoC-Editionen. Im Zusammenhang mit Beratung und Psychotherapie nimmt die vorliegende Leitlinie Bezug auf die von der American Psychological Association (2015) veröffentlichten „Guidelines for Psychological Practice with Transgender and Gender Nonconforming People“. Formuliert werden 16 Empfehlungen für eine affirmative psychotherapeutische Praxis (siehe Kapitel 5 der Leitlinie; DGfS 2018: 37 f.). In einer Stellungnahme zu den Transgender People fordert die World Medical Association (2015) das Selbstbestimmungsrecht der Menschen über ihr Geschlecht. Nicht nur behandelnde Ärzt_innen und Psychotherapeut_innen, sondern auch Regierungen und staatliche Organisationen werden aufgefordert, adäquate Behandlungsangebote in der Gesundheitsversorgung einzurichten, die diskriminierungsfrei die Individualität des Einzelnen respektieren.

Zusammengenommen stehen die internationalen Empfehlungen für einen State of the Art, der sich wie folgt beschreiben lässt: Trans-Identitäten sind nicht pathologisch, behandelt wird die Geschlechtsinkongruenz sensu ICD-11 (Klein et al. 2015; World Health Organisation 2018) und/oder die Geschlechtsdysphorie sensu DSM-5 (American Psychiatric Association 2013; Zucker et al. 2013). Trans Menschen soll ein verlässlicher Zugang zu multimodaler transitionsunterstützender Gesundheitsversorgung möglich sein (Reisner et al. 2016). Unter Berücksichtigung des Körpers, der Psyche und der sozialen Situation sollen trans Menschen im Einklang mit ihrem Geschlecht leben können. Dabei steht die Gesundheitsversorgung für trans Menschen im Vordergrund, die offen ist für Menschen, die ihr Geschlecht binär empfinden (männlich vs. weiblich) sowie für diejenigen, die es non-binär empfinden (z. B. agender, geschlechtsneutral, genderqueer). In Abkehr von den vormals rigiden und überregulierten Behandlungen (Hamm und Sauer 2014; Nieder et al. 2014a) wird ein individualisiertes und flexibles Vorgehen im Zuge einer möglichen Transition gefordert. Es soll sich nicht mehr um eine Begutachtung und Auswahl geeigneter Kandidat_innen für somatische, geschlechtsangleiche Behandlungen handeln (Garcia Nuñez und Nieder 2017), die Funktion eines Gatekeepers soll vermieden werden (Hamm und Sauer 2014: 27; Richards et al. 2014). Es gibt keine absoluten Ausschlusskriterien für transitionsunterstützende somatische Maßnahmen, d. h. es gibt keine einzelnen Kriterien, die eine somatische Behandlung zur Geschlechtsangleichung automatisch und bedingungslos ausschließen. In einem relativen Sinne gibt es solche Kriterien durchaus, z. B. aus situativen, kontextuellen oder zeitgebundenen Gründen. Das Ziel der Behandlung ist die Reduktion der Geschlechtsinkongruenz und des mit der Geschlechtsinkongruenz einhergehenden Leidensdrucks, der Geschlechtsdysphorie (Nieder et al. 2016).
Die vorliegende Arbeit schließt an den ersten Artikel zur Leitlinienentwicklung von Nieder und Strauß (2014) an und beschreibt aus methodischer Sicht ihren weiteren Verlauf.

\section{Methoden der S3-Leitlinie: Von der Evidenzbasierung zur Konsentierung}

Die AWMF definiert mit S3 eine Leitlinie, bei deren Entwicklung eine Reihe von methodischen Kriterien zu beachten ist (AWMF 2012). Die Literaturrecherche muss hierfür einer standardisierten Methode folgen, sollte detailliert beschrieben sein (inkl. Auswahlkriterien und Ausschlussgründen) und nach zuvor festgelegten Kriterien ob ihrer methodischen Qualität bewertet werden. Die systematische Recherche orientierte sich daher an den Vorgaben der AWMF bzw. des Deutschen Instruments zur methodischen Leitlinien-Bewertung (DELBI; Ärztliches Zentrum für Qualität in der Medizin 2008). Die Auswahl und Bewertung wissenschaftlicher Evidenz erfolgte nach den Qualitätskriterien des Scottish Intercollegiate Guidelines Network (SIGN). Zur Formulierung der Empfehlungen wurden formale Konsensustechniken angewendet und jede Empfehlung wurde im Zuge einer strukturierten Konsensfindung diskutiert und abgestimmt.

\section{Systematische Literaturrecherche}

Im Vordergrund der systematischen Literaturrecherche stand die interventionsbezogene Primärliteratur, d. h. gesucht wurden empirische Studien, die die Wirksamkeit von Interventionen im Behandlungsfeld untersucht haben. Die Suche ${ }^{3}$ erfolgte über die Datenbanken Medline, PsycINFO und Web of Science. Gesucht wurde Literatur aus dem Zeitraum zwischen 1990 und 2014. Die Suchbegriffe wurden von der Steuerungsgruppe im Anschluss an existierende Suchen aus Metaanalysen (Murad et al. 2010; Sutcliffe et al. 2009) ausgewählt. Die Suchstrategie wurde hinsichtlich ihrer Sensitivität (möglichst geringe Anzahl nicht gefundener relevanter Referenzen) und Spezifität (möglichst geringe Anzahl gefundener irrelevanter Referenzen) geprüft und ausgewertet. Eine möglichst große Sensitivität wurde einer großen Spezifität vorgezogen. Inhaltlich ging es bei der Suche vor allem um die Fragen, ob und wenn ja, zu welchen Voraussetzungen (bspw. wie von der Begutachtungsrichtlinie gefordert; MDS 2009) und zu welchen Behandlungen empirische Wirksamkeitsnachweise vorliegen.

\section{Auswahl der Evidenz}

Die Datenbanksuche ergab 5437 Treffer. Nach einer Prüfung auf Relevanz (1. Screening) blieben 4341 Referenzen übrig, von denen nach Prüfung der Volltexte (2. Screening) 448 Studien berücksichtigt wurden. Ausgeschlossen wurden Arbeiten, die sich (I) nicht auf die Trans-Thematik beziehen, die (II) nicht empirisch sind, die sich (III) ausschließlich auf Kinder und Jugendliche beziehen ${ }^{4}$, die

3 Im Leitlinienreport(S.4 f., s. www.awmf.org/leitlinien/detail/II/138-001. html) findet sich exemplarisch der Suchstring für Medline. Für die anderen Datenbanken wurde die Suchstrategie inhaltsgleich angewandt.

4 Eine entsprechende Leitlinie für Kinder und Jugendliche wird derzeit unter Federführung der Deutschen Gesellschaft für Kinder- und Jugendpsychiatrie, Psychosomatik und Psychotherapie (DGKJP) entwickelt. 
- Tab. 1 Klassifikation der Evidenz.

\begin{tabular}{|c|l|}
\hline I & $\begin{array}{l}\text { Metaanalysen, systematische Übersichtsarbeiten über methodisch } \\
\text { hochwertige Studien, randomisiert-kontrollierte Studien. }\end{array}$ \\
\hline II & $\begin{array}{l}\text { Systematische Übersichtsarbeiten über methodisch weniger } \\
\text { hochwertige Studien, Kohortenstudien oder Fall-Kontroll-Studien } \\
\text { mit geringem Verzerrungspotential und einer Wahrscheinlichkeit, } \\
\text { dass der gefundene Zusammenhang kausal bedingt ist. }\end{array}$ \\
\hline III & $\begin{array}{l}\text { Kohortenstudien oder Fall-Kontroll-Studien mit } \\
\text { Verzerrungspotential, Fallberichte. }\end{array}$ \\
\hline
\end{tabular}

(IV) keinen Bezug zu einem psychosozialem Outcome haben, die sich (V) ausschließlich auf Aspekte der Ätiologie beziehen, die (VI) sprachlich für die Mitglieder der Leitliniengruppe nicht verwertbar sind (z. B. Arbeiten in portugiesischer oder hebräischer Sprache) und solche, die (VII) vor 1990 veröffentlicht wurden. Der Zeitpunkt wurde gewählt, weil die ICD-10 im Jahr 1990 veröffentlicht und das fokussierte Thema als Geschlechtsidentitätsstörung neu konzeptualisiert wurde (vorher: sexuelle Deviation). Zudem gab es erstmals operationalisierte Forschungskriterien. Insgesamt erfolgte die Auswahl der Evidenz durch die Mitglieder der Steuerungsgruppe in zwei Schritten (AWMF 2012).

\section{Evidenzbewertung}

Die systematisch recherchierte und selektierte Literatur wurde mithilfe einer Vorlage zur Evidenzbewertung geprüft. Berücksichtigt wurden jeweils das Studiendesign, die Stichprobe(n), die Durchführung und die Auswertung der Studien. Zusätzlich wurden klinische Aspekte der Studien berücksichtigt, z. B. inwiefern die Stichprobe der Heterogenität von trans Personen entspricht und die Vergleichsgruppen für die Beantwortung der Fragestellung angemessen ausgewählt wurden. Bei Katamnesestudien wurde geprüft, ob die Endpunkte klinisch relevant waren und ob der Zeitraum zur Nachbeobachtung ausreichend lang bzw. die Zahl der inkludierten Teilnehmenden ausreichend groß war, um mögliche Effekte zu finden. Auch das Verzerrungspotential einer Studie wurde bei der Evidenzbewertung berücksichtigt (vgl. Hrobjartsson et al. 2013). Zur Klassifikation der Evidenz wurde eine dreiteilige Evidenzhierarchie angewendet (I-III) ( Tab. 1)

\section{Formulierung der Empfehlungen}

Die Empfehlungen zur Behandlung sind entweder empirisch fundiert und belegen dies mit der Angabe von Quellen, deren methodische Qualität bewertet wurde, oder sie resultieren aus den klinisch-therapeutischen Erfahrungen der beteiligten Mandatsträger_innen, wenn zu der Aussage keine wissenschaftliche Evidenz vorlag. Bei der Formulierung der Empfehlungen wurden die Vorgaben des AWMF-Regelwerks angewandt (2012): Die Verwendung von „soll“ bringt eine starke Empfehlung zum Ausdruck, „sollte“ reflektiert eine Empfehlung. Eine offene Empfehlung wird über die Formulierung „kann“ ausgedrückt. Die evidenzbasierten Empfehlungen stellen jeweils die zugrunde liegende Literatur und den Evidenzgrad dar (i. d. R. Evidenzgrad III). Zudem stehen Evidenztabellen zur allgemeinen Wirksamkeit körpermodifizierender Behandlungen, zur Hysterektomie und zur Neovagina unter www.awmf. org/leitlinien/detail/II/138-001.html separat zur Verfügung.

\section{Formale Konsensfindung}

Die Konsensfindung zu den Behandlungsempfehlungen fand im Rahmen von drei Konsensuskonferenzen im Frühjahr, Sommer und Winter 2016 in Berlin statt. Anwesend waren jeweils die Mandatsträger_innen der beteiligten Fachgesellschaften ${ }^{5}$ sowie Vertreter_innen von Berufs- ${ }^{6}$ und Interessensverbänden. ${ }^{7}$ Jede Empfehlung wurde im Zuge einer formalen Konsensustechnik, dem nominalen Gruppenprozess (NGP), diskutiert und unter neutraler Moderation der AWMF konsentiert ${ }^{8}$.

\section{Aufbau der Leitlinie}

Die Leitlinie gliedert sich in acht Kapitel. Nach der „Einführung“ (1), die sich mit der Terminologie, der Ausgangs- und Versorgungssituation, den Diagnosen, der Epidemiologie, den Zielen und der Zielgruppe sowie mit dem Aufbau und der Methodik der Leitlinie befassen, folgen ein Abschnitt zur Situation von „Trans Personen im Gesundheitssystem“ (2) und zur „Therapeutische(n) Haltung und Beziehungsgestaltung“ (3). Im Anschluss fokussiert ein Kapitel (4) die „Diagnostik“ und leitet zum Thema „Beratung und Psychotherapie“ (5) über. Zur „Vorbereitung körpermodifizierender Behandlungen“ (6) werden Outcome-Studien berichtet, die im folgenden Abschnitt mit Blick auf die verschiedenen „körpermodifizierenden Behandlungen“ (7) spezifiziert werden (Hormone, Epilation, Brustbereich, Genitalbereich, Adamsapfel, gesichtsfeminisierende Operationen, Hilfsmittel). Das Abschlusskapitel thematisiert relevante Fragen zur „Trans-Gesundheit“ (8) im Zusammenhang mit Transition und Re-Transition, Sexualität und Intimität, Familie und Arbeit.

\section{Öffentliche Kommentierung}

Im Frühjahr 2017 stand eine vorläufige Version der Leitlinie für vier Wochen online zur Kommentierung für die interessierte Öffentlichkeit bereit. Der Link wurde über das Netzwerk des Bundesverbands Trans* (BVT*) verbreitet. Die Online-Umfrage wurde insgesamt 3485-mal aufgerufen, davon allerdings in 2794 Fällen bis zur dritten Seite abgebrochen. An der Umfrage beteiligt haben sich schließlich N=1401 Personen, von denen wiederum 378 Personen die Kommentierung vollständig abgeschlossen haben. Im Mittel haben die Kommentator_innen 54 Minuten benötigt. Die überwiegende Mehrheit der Kommentare war positiv (z. B. „vielen Dank für eure tolle Arbeit!!!“, „sehr gut :-)“, „Volle Zustimmung“,

5 Deutsche Gesellschaft für Sexualforschung (DGfS), Akademie für Ethik in der Medizin (AEM), Deutsche Ärztliche Gesellschaft für Verhaltenstherapie (DÄVT), Deutsche Gesellschaft für Medizinische Psychologie (DGMP), Deutsche Gesellschaft für Psychiatrie und Psychotherapie, Psychosomatik und Nervenheilkunde (DGPPN), Deutsche Gesellschaft für Psychoanalyse, Psychotherapie, Psychosomatik und Tiefenpsychologie (DGPT), Deutsche Gesellschaft für Psychosomatische Medizin und Ärztliche Psychotherapie (DGPM), Deutsches Kollegium für Psychosomatische Medizin (DKPM), Gesellschaft für Sexualwissenschaft (GSW), Schweizerische Gesellschaft für Psychiatrie und Psychotherapie (SGPP), Deutsche Gesellschaft für Sexualmedizin, Sexualtherapie und Sexualwissenschaft (DGSMTW).

6 Berufsverband Deutscher Nervenärzte (BVDN), Berufsverband Deutscher Psychiater (BVDP), Bundespsychotherapeutenkammer (BPtK).

7 Bundesvereinigung Trans* (BVT*).

8 Weitere Details zum Verfahren des NGP und der Konsentierung finden sich im Leitlinienreport (S. 7; s. www.awmf.org/leitlinien/detail/II/138001.html). 
„Einverstanden“). Kritische Kommentare bezogen sich häufig nicht konkret auf die Statements, sondern waren allgemein gehalten („Es wird ein unnötiges Monopol geschaffen mit Argumentationen aus den 1970er Jahren. Es wird evidenzbasiertes Arbeiten postuliert, aber nirgendwo gemacht.“, „Ohne psychische Störung braucht es keine Empfehlungen.“, „Ich halte solche Empfehlungen für einen Fehler“). Darüber hinaus boten die Kommentare inhaltliche und strukturelle Kritik an den Empfehlungen, die sich direkt an den Kapiteln orientierte. Die vorgeschlagenen Änderungen, die sich aus der Online-Kommentierung ergeben hatten, wurden im Rahmen der Steuerungsgruppe diskutiert und ihre Berücksichtigung für die Leitlinie befürwortet oder abgelehnt. ${ }^{9}$ Die Änderungen, die für einzelne Empfehlungen resultierten, wurden bei der dritten Konsensuskonferenz konsentiert. Im Anschluss wurde die Leitlinie an die Vorstände der beteiligten Fachgesellschaften zur Konsentierung weitergeleitet. Eine Konsentierung erfolgte durch alle Gesellschaften mit Ausnahme der DGSMTW, die stattdessen eine kritische Stellungnahme in den Umlauf brachte (siehe hierzu später im Text). Die Stellungnahme und eine Entgegnung der Leitliniengruppe finden sich im Leitlinienreport auf der AWMF-Seite.

\section{Zentrale Behandlungsempfehlungen und ihre Bedeutung}

Der folgende Abschnitt stellt zentrale Behandlungsempfehlungen vor, um anhand dessen die inhaltliche Ausrichtung der Leitlinie zu verdeutlichen.

\section{Partizipative Entscheidungsfindung}

Aus dem Kapitel „Therapeutische Haltung und Beziehungsgestaltung“ (3) ist die folgende Empfehlung von Bedeutung (DGfS 2018: 18):

\begin{abstract}
„Entscheidungen über die Notwendigkeit und die Reihenfolge der Behandlungsschritte sollen partizipativ im Sinne einer Übereinstimmung zwischen Behandlungssuchenden und Behandelnden getroffen werden. Sollte im Einzelfall eine Übereinstimmung nicht herstellbar sein, so sollten die Gründe dafür transparent dargelegt werden (Konsensbasierte Empfehlung, Konsensstärke: Konsens).“
\end{abstract}

Die Empfehlung folgt dem Ansatz der partizipativen Entscheidungsfindung. Allerdings ist die Empfehlung aus Sicht der BVT* (Bundesverband Trans*) beschönigend, da letztlich nichts passiere, wenn der_die Behandler_in nicht zustimmt. Die behandlungssuchende Person erhalte nicht die notwendige Prokura, um eigenverantwortlich ihre Transition zu gestalten. Da die BVT* diese Empfehlung nicht mittragen konnte, hat sie die Aufnahme eines Sondervotums beantragt (AWMF 2012) und einen Alternativvorschlag formuliert, um den Dissens offen zu legen (DGfS 2018: 18):

9 Eine tabellarische Auflistung aller Vorschläge inklusive der Begründung für oder gegen eine Aufnahme kann beim Erstautor angefordert werden.
„Wenn eine trans Person einwilligungsfähig ist und den Umfang und die Konsequenzen der Behandlung verstanden hat, soll die informierte Entscheidung im Sinne eines Informed Consents über die Notwendigkeit und die Reihenfolge der Behandlungsschritte allein bei ihr liegen (Sondervotum Bundesverband Trans*).“

Mit dem Sondervotum befürwortet die BVT* ein Modell, das die Entscheidungshoheit über Notwendigkeit und Reihenfolge der einzelnen transitionsunterstützenden Behandlungen ausschließlich bei der trans Person sieht. International gibt es für diese Vorgehensweise bereits Vorbilder (Deutsch 2012; Radix und Eisfeld 2014), in Deutschland hat sie sich bisher nicht durchgesetzt. Aus Sicht der Leitlinienkoordination ist das Sondervotum daher sinnvoll: Es markiert einen zentralen Dissens, der seit vielen Jahren in diesem Versorgungsfeld besteht und sich bis dato nicht lösen lässt (Hamm und Sauer 2014; Nieder und Strauß 2014).

\section{Diagnostik}

Im Kapitel zur „Diagnostik“ (4) führt die Leitlinie zunächst ein, welche Bereiche die der Diagnostik zugrunde liegende Anamnese allgemein umfassen soll (DGfS 2018: 25):

„Im Rahmen der Diagnostik und individuellen Behandlungsplanung soll eine Anamneseerhebung erfolgen, die die psychosexuelle Entwicklung, Sozialanamnese, biographische Anamnese und medizinische Anamnese umfasst, sowie der psychische Befund erhoben werden (Konsensbasierte Empfehlung, Konsensstärke: Konsens).“

Im Anschluss konkretisiert eine Empfehlung, was die Anamnese evidenzbasiert beinhalten sollte (DGfS 2018: 25f):

„Geschlechtsinkongruenz vor der Pubertät, Pubertätsentwicklung, Geschlechtsidentität, Partnerschaften, Finden von Selbstbezeichnungen, Coming-out, Erfahrungen von Stigmatisierung und Diskriminierung, familiäre und gesellschaftliche Einflüsse, bisherige Erfahrungen im Gesundheitssystem, selbst angestrebte Maßnahmen zur Reduktion der Geschlechtsdysphorie, Ressourcen (Gomez-Gil et al. 2014; Hawkins 2009; Kenagy und Hsieh 2005; Kuper et al. 2012; Monro 2000; Nuttbrock et al. 2013; Poteat et al. 2013; Schulz 2012; Xavier et al. 2005; Yerke und Mitchell 2011) (Evidenzbasierte Empfehlung: Evidenzgrad III, Abstimmung der Empfehlung: Konsens).“

Schließlich wird empfohlen, die Dauer der geschlechtsbezogenen Symptomatik zu erfassen (DGfS 2018: 26):

„Es sollte erfasst werden, ob die Geschlechtsinkongruenz und/ oder die Geschlechtsdysphorie konstant zumindest seit einigen Monaten bestehen, vorübergehend oder intermittierend ist (Konsensbasierte Empfehlung, Konsensstärke: Starker Konsens).“ 


\section{Differentialdiagnostik}

Im Zusammenhang mit der Diagnostik einer Geschlechtsinkongruenz und/oder Geschlechtsdysphorie (GIK/GD) ist auch die Differentialdiagnostik von Bedeutung. Allerdings gelten Diagnosen, die einen Transsexualismus noch ausgeschlossen hätten (z. B. psychotische Störungen), nicht mehr unmittelbar als Ausschlusskriterium für eine GIK/GD, wie die folgende Empfehlung verdeutlicht (DGFS 2018: 31):

„Bei jeder in Frage kommenden Differentialdiagnose besteht die Möglichkeit, dass es sich lediglich um eine zusätzliche Diagnose handelt. Sie schließt insofern nicht automatisch eine Geschlechtsinkongruenz und/oder Geschlechtsdysphorie aus (Konsensbasiertes Statement, Konsensstärke: Starker Konsens).“

Das bedeutet jedoch keineswegs, dass begleitende psychische Störungen unbeachtet bleiben sollen, im Gegenteil. Die folgenden Empfehlungen illustrieren das Vorgehen, das die Leitlinie für den Umgang mit psychischen Störungen vorsieht. Evidenzbasiert ist die folgende Aussage (DGfS 2018: 35):

„Ein bedeutsamer Anteil der Behandlungssuchenden hat keine psychischen Störungen (De Cuypere et al. 1995; Dhejne et al. 2016; Gomez-Gil et al. 2009; Hepp et al. 2005; Heylens et al. 2014; Levine und Solomon 2009) (Evidenzbasiertes Statement, Evidenzgrad III).“

Evidenzbasiert sind allerdings auch die zwei folgenden Empfehlungen, die im Zusammenhang mit psychotischen Störungen von Bedeutung sind. Zunächst geht es um das Risiko, das von nicht diagnostizierten oder nicht adäquat behandelten psychotischen Störungen im Zuge einer Transition ausgehen kann (DGfS 2018: 35):

„Bei nicht diagnostizierten oder nicht adäquat behandelten psychotischen Störungen kann es zum Bedauern der Entscheidung für modifizierende Behandlungen der körperlichen Geschlechtsmerkmale kommen (Borras et al. 2007; Campo et al. 2001; Commander und Dean 1990; jiloha et al. 1998) (Evidenzbasierte Empfehlung: Evidenzgrad III, Konsensstärke: Starker Konsens).“

Auch wird der empfohlene Umgang mit psychotischen Störungen beschrieben (DGfS 2018: 36):

„Psychotische Störungen sollen zunächst einer adäquaten Therapie zugeführt werden, bevor Geschlechtsinkongruenz und/oder Geschlechtsdysphorie diagnostiziert wird (Borras et al. 2007; Campo et al. 2001; Caspari et al. 1999; Commander und Dean 1990; jiloha et al. 1998) (Evidenzbasierte Empfehlung: Evidenzgrad III, Konsensstärke: Starker Konsens).“

Eine Behandlungsempfehlung gibt es zudem allgemein für begleitende psychische Störungen, die eine Behandlung der GIK/GD allerdings nicht ausschließt (DGfS 2018: 36):
„Sowohl reaktive als auch unabhängige psychische Störungen sollten bis zur Remission parallel zur Behandlung der Geschlechtsdysphorie adäquat psychotherapeutisch und/oder psychopharmakologisch behandelt werden (Konsensbasierte Empfehlung, Konsensstärke: Starker Konsens).“

Mit den dargestellten Empfehlungen soll ein Rahmen geschaffen werden, in dem einerseits die Behandlung der GIK/GD auch parallel zur Behandlung begleitender psychischer Störungen zielführend möglich ist, d. h. mit Fokus auf die Reduktion der GIK/GD, und andererseits - insbesondere beim Vorliegen psychotischer oder schwerer dissoziativer Störungen - erst eine adäquate Behandlung der entsprechenden Erkrankung erfolgen soll, bevor körpermodifizierende Maßnahmen zur Behandlung der GIK/GD erwogen werden.

\section{Voraussetzungen für den Beginn somatischer Behandlungen}

Dieser Abschnitt stellt eine zentrale Änderung zu dem bisherigen Vorgehen in der Behandlung dar. Die Optionen für eine Behandlung der GIK/GD werden aufgezeigt und daran anschließend wird empirisch sowie klinisch fundiert ein Vorgehen empfohlen, das auf individuelle Bedingungen bei der Behandlung bedarfsgerecht eingehen soll. So werden Psychotherapie und die so genannten Alltagserfahrungen nicht mehr als notwendige Voraussetzungen für bspw. eine Hormonbehandlung betrachtet. Konsensbasiert wird empfohlen (DGfS 2018: 45):

„Psychotherapie soll nicht ohne spezifische Indikation angewandt und keinesfalls als Voraussetzung für körpermodifizierende Behandlungen gesehen werden. Die Indikation ist nach den Vorgaben der Psychotherapierichtlinie zu stellen (Konsensbasierte Empfehlung, Konsensstärke: Konsens).“

Allerdings kann eine Psychotherapie hilfreich sein (Löwenberg und Ettmeier 2014), sodass evidenzbasiert empfohlen wird (DGfS 2018: 44):

„Psychotherapie kann zur Minderung der Geschlechtsdysphorie (z. B. durch eine Förderung der Selbstakzeptanz, der Bewältigung negativer Gefühle und eine Unterstützung bei der Identitätsentwicklung) beitragen (Bockting et al. 2007; Byne et al. 2012) (Evidenzbasiertes Statement: Evidenzgrad III, Konsensstärke: Starker Konsens).“

Mit Blick auf die Ziele einer Psychotherapie wird zudem ergänzt (DGfS 2018: 45):

„Psychotherapie soll trans Personen im Bedarfsfall angeboten werden. Ziele einer Psychotherapie können sein: Förderung von Selbstakzeptanz, Selbstwertgefühl und Selbstsicherheit, Bewältigung von Scham- und Schuldgefühlen sowie von internalisierter Trans-Negativität, Unterstützung der Identitätsentwicklung, Reflexion und Bearbeitung möglicher Erfahrungen und Konflikte in einer anderen Geschlechtsrolle, Unterstützung des Coming-Out-Prozesses, insbesondere bei familiären oder partnerschaftlichen Problemen, Unterstützung bei an- 
deren familiären oder partnerschaftlichen Problemen, Unterstützung bei Problemen im Zusammenhang mit der eigenen Elternrolle, Bearbeitung der Auswirkungen der Reaktionen anderer (Trans-Negativität und Trans Feindlichkeit), Unterstützung bei einer Entscheidung über körpermodifizierende Behandlungen, Unterstützung nach körpermodifizierenden Behandlungen, Unterstützung bei andauernder Geschlechtsdysphorie (Konsensbasierte Empfehlung, Konsensstärke: Starker Konsens).“

Das anhand der Empfehlungen zur Psychotherapie illustrierte Vorgehen der Leitlinie zeigt sich ähnlich auch bei der Frage nach Alltagserfahrungen (DGfS 2018: 47):

„Alltagserfahrungen mit dem Wechsel von der bisherigen Geschlechtsrolle in eine andere stellen keine notwendige Voraussetzung für den Beginn körpermodifizierender Behandlungen zur Unterstützung einer Transition dar (Konsensbasierte Empfehlung, Konsensstärke: Starker Konsens).“

Allerdings stellt die Leitlinie auch den potentiellen Nutzen von Alltagserfahrungen heraus und empfiehlt konsensbasiert das Folgende (DGfS 2018: 47):

\begin{abstract}
„Alltagserfahrungen, bei denen sich Behandlungssuchende in der gewünschten Rolle erfahren, können aufschlussreich für die weitere Transition, für Entscheidungen für oder gegen transitionsunterstützende Behandlungen sowie für die Wahl der geeigneten Zeitpunkte sein (Konsensbasierte Empfehlung, Konsensstärke: Konsens).“
\end{abstract}

Abgeschlossen wird das Thema von einer evidenzbasierten Aussage, die diverse Alltagserfahrungen empfiehlt (DGfS 2018: 47):

„Für eine voll informierte soziale und medizinische Transition sind möglichst vielfältige Alltagserfahrungen zu empfehlen. Es sollte sich um ein individualisiertes Vorgehen handeln, das bei Vorliegen einer Indikation mit Beginn bspw. einer Hormonbehandlung, einer Entfernung der Gesichtsbehaarung oder einer Mastektomie einhergehen kann. Rücksicht genommen werden sollte auf Diskriminierungspotentiale (Barker und Wylie 2008; Ellis et al. 2014) (Evidenzbasiertes Statement: Evidenzgrad III, Konsensstärke: Konsens). “

Es folgen die transitionsunterstützenden somatischen Behandlungen, deren Bedeutung im Zuge der Behandlung der GIK/GD unterstrichen wird (DGfS 2018: 52):

„Modifizierende Behandlungen körperlicher Geschlechtsmerkmale sind für trans Personen, die körpermodifizierende Behandlungen in Anspruch nehmen wollen, die Therapie der ersten Wahl (Konsensbasierte Empfehlung, Konsensstärke: Starker Konsens).“

Weder Psychotherapie noch Alltagserfahrungen werden als unbedingt notwendige Voraussetzungen für den Beginn von somatischen Behandlungen bei GIK/GD gefordert. Die Leitlinie verzich- tet auf zeitliche Fristen, die von den Behandlungssuchenden erfüllt werden sollen. So sind bspw. die Behandlung mit Sexualhormonen (DGfS 2018: 57) und die maskulinisierende Operation im Brustbereich (DGfS 2018: 62) an den Abschluss der Diagnostik gekoppelt:

„Die Hormontherapie soll nach Abschluss der Diagnostik ermöglicht werden (Konsensbasierte Empfehlung, Konsensstärke: Konsens).“

„Die maskulinisierende Operation im Brustbereich soll den Behandlungssuchenden nach Abschluss der Diagnostik ermöglicht werden (Konsensbasierte Empfehlung, Konsensstärke: Starker Konsens).“

Auch die Entfernung der Gesichtsbehaarung kann zu Beginn der Transition möglich sein (DGfS 2018: 60):

„Die Epilation der Gesichtsbehaarung soll den Behandlungssuchenden bereits zu Beginn der Transition ermöglicht werden (Konsensbasierte Empfehlung, Konsensstärke: Starker Konsens).“

Andere Behandlungen sollen sich an vorherige Maßnahmen anschließen, z. B. die Mammaplastik (DGfS 2018: 63):

„Behandlungssuchenden soll nach Ausschöpfung hormoneller Möglichkeiten eine Mammaplastik ermöglicht werden (Konsensbasierte Empfehlung, Konsensstärke: Starker Konsens).“

Oder ein phonochirurgischer Eingriff (DGfS 2018: 69):

„Nach Ausschöpfen logopädischer Behandlungen sollte den Behandlungssuchenden ein phonochirurgischer Eingriff ermöglicht werden (Konsensbasierte Empfehlung, Konsensstärke: Starker Konsens).“

\section{Diskussion}

Mit einem schnellen Blick kann der Leitlinie bei der Indikationsstellung für somatische Behandlungen ein unkritisches Vorgehen zugeschrieben werden, wenn unterstellt wird, wie von der DGSMTW (2018: 3) geschehen, dass „stets alles allen Behandlungssuchenden ,ermöglicht werden““ soll. So fehlt der DGSMTW eine „medizinisch notwendige Binnendifferenzierung“, der „Verzicht auf jegliche Alltags-Erprobung“ sei „besonders problematisch“ (DGSMTW 2018: 3). Allerdings geht weder das Vorliegen der ICD-11-Diagnose Geschlechtsinkongruenz noch das der DSM-5-Diagnose Geschlechtsdysphorie unmittelbar mit der Indikation einzelner körperverändernder Behandlungen einher (Nieder et al. 2016). Zur Indikationsstellung braucht es ein individuell fundiertes, d. h. am Einzelfall orientiertes und klinisch begründetes Vorgehen, für das die Leitlinie einen Behandlungsrahmen bereitstellen will. Ein solches Vorgehen muss allerdings als Bedingung guter klinischer $\mathrm{Ar}$ beit nicht expressis verbis von einer S3-Leitlinie ausgewiesen werden. Bedeutsam ist hier die bereits vorgestellte Empfehlung, dass die Entscheidungen über die Notwendigkeit und die Reihenfolge der Behandlungsschritte partizipativ im Sinne einer Übereinstim- 
mung zwischen Behandlungssuchenden und Behandelnden getroffen werden sollen. Behandelnde sollen mögliche Zweifel gegenüber einer Indikation für eine somatische Behandlung nicht mehr mit den Vorgaben der Begutachtungsrichtlinie (MDS 2009) kaschieren („Die Krankenkasse will das so...“). Vielmehr sollen sie ihre Zweifel den Behandlungssuchenden gegenüber verständlich vermitteln, sodass bestenfalls gemeinsam oder im Rahmen einer verteilten Behandlung an den möglichen Problemen oder Zweifeln gearbeitet werden kann (Laszig et al. 1995).

Die skizzierten Bereiche Psychotherapie und Alltagserfahrungen bzw. die dort jeweils aufeinander folgenden Empfehlungen illustrieren exemplarisch die dialektische Ausrichtung der Leitlinie. Zunächst wird eine bislang als zwingend formulierte Voraussetzung für somatische Behandlungen (Psychotherapie, Alltagserfahrungen) bzw. ihr Ausschluss (psychische Störungen) auf empirische Wirksamkeit geprüft, die jeweils nicht ausreichend gegeben war. Weder Psychotherapie noch Alltagserfahrungen sind demnach zwingend notwendig. Die Leitlinie ist klar in ihrer Position zur Deregulierung. Kritisch berücksichtigt werden in diesem Zusammenhang ethische Fragen zur Autonomie und Selbstbestimmung sowie therapeutische Überlegungen hinsichtlich der möglichen Inkaufnahme von schädlichen Effekten von Diskriminierungserfahrungen. Beachtet werden allerdings auch die klinischen Erfahrungen der Mandatsträger_innen, nach denen sowohl Psychotherapie als auch Alltagserfahrungen für die Transition hilfreich sein können sowie die empirischen Befunde, nach denen Psychotherapie unter bestimmten Voraussetzungen und die Alltagserfahrung zu empfehlen (Bockting 2008) und teils gewünscht (Nieder und Strauss 2015) sind. Beide Aspekte können daher für eine individuelle Behandlung sehr bedeutsam sein.

\section{Limitationen}

Die Leitliniengruppe hatte zu Beginn der Leitlinienentwicklung beschlossen, einen psychosozialen Schwerpunkt zu setzen. Demzufolge äußert sich die S3-Leitlinie nicht zur technischen Umsetzung einzelner somatischer Behandlungen (z. B. chirurgische Eingriffe). Zu den geschlechtsangleichenden chirurgischen Maßnahmen hat sich zu Beginn dieses Jahres eine Leitliniengruppe unter Federführung der Deutschen Gesellschaft für Urologie (DGU) und der Deutschen Gesellschaft der Plastischen, Rekonstruktiven und Ästhetischen Chirurgen (DGPRÄC) zusammengefunden, die anstrebt, eine Leitlinie auf S2k-Niveau zu entwickeln (AWMF-Registernummer: 043-052). Beide Fachgesellschaften hatten sich positiv zur S3-Leitlinie geäußert. Empfehlungen zur Hormontherapie bei GIK/GD liegen international vor (Hembree et al. 2017), eine AWMF-Leitlinie zur Hormonbehandlung liegt nicht vor.

Ebenfalls sollte einschränkend erwähnt sein, dass aufgrund des eingeschränkten Suchzeitraums - vom 1.1.1990 bis zum 23.6.2014 - Studien, die ab der zweiten Jahreshälfte 2014 erschienen sind, für die Evidenzbewertung nicht berücksichtigt wurden.

\section{Fazit und Ausblick}

Die hier beschriebene Leitlinie verfolgt einen ganzheitlichen Ansatz und stellt die Transition in einen direkten (Kapitel 5, 6, 7) und indirekten (Kapitel 2, 3, 4, 8) Zusammenhang mit der Gesundheit von trans Personen. Bei der Diagnostik bezieht sie sich auf Katego- rien der ICD-11 und des DSM-5. Damit einhergehend ist sie offen für alle Menschen, für die diese Diagnosen infrage kommen, und damit auch für Behandlungssuchende, die sich divers, non-binär, agender und/oder genderqueer definieren (Koehler et al. 2018). Zudem kommen die Empfehlungen zu den somatischen Behandlungen ohne zeitliche Kriterien aus, weder Psychotherapie noch Alltagserfahrungen werden als unbedingt notwendige Voraussetzungen gefordert. Im Zusammenhang mit der Indikation somatischer Behandlungen spielen Aspekte der informierten Zustimmung und der partizipativen Entscheidungsfindung eine zunehmende Rolle. Damit hält die Leitlinie Schritt mit den in der Einleitung skizzierten internationalen Entwicklungen und dem daraus folgenden State of the Art. Die Behandler_innen sollen sich auf ihre klinisch-therapeutische Expertise besinnen und sich darauf konzentrieren können, was den Behandlungssuchenden mit Blick auf die Reduktion der GIK/GD und die Erhöhung der Lebensqualität hilft. Letztlich sollen die Behandelnden durch die Leitlinie ermutigt werden, ihre klinische Expertise frei von zeitlichen Vorgaben („ein Jahr Psychotherapie“) einzusetzen und mit offenen Fragen bei der Indikationsstellung gegenüber den Betreffenden transparent umzugehen. Die Anwendung der Leitlinie sollte mit klinisch-therapeutischer Expertise abgesichert werden, ein differenziertes und am Einzelfall orientiertes Vorgehen soll gleichermaßen Ziel und Folge der Leitlinie sein.

Die ersten Rückmeldungen zur Rezeption der Leitlinie sind ambivalent. Zunächst wurden uns Freude und Erleichterung darüber entgegengetragen, dass die Leitlinienentwicklung nun vorerst abgeschlossen werden konnte. Allerdings scheinen der Wegfall von zeitlich vorgegebenen Fristen und dem damit einhergehenden, in der Regel erwarteten Behandlungsablauf sowie die Öffnung für nonbinäre Behandlungssuchende zu verunsichern. Während zu Zeiten der Behandlungsstandards nach Becker et al. (1997) die Verunsicherung und die Ambivalenzen eher aufseiten der Behandlungssuchenden zu verorten waren, haben die Affekte nun womöglich die Seiten gewechselt.

Um die Verunsicherung und die Ambivalenzen für eine bedarfsgerechte Behandlung nutzen zu können, braucht es im Kontakt mit den Behandlungssuchenden einen reflektierten und bewussten Umgang mit den eigenen, auch ambivalenten Affekten und eine Konzentration auf sorgfältige klinische Arbeit. Eine detaillierte Anamnese, eine fundierte Diagnostik, eine belastbare Dokumentation, eine tragfähige Behandlungsplanung sowie ein offenes InBeziehung-Treten brauchen Zeit, Akzeptanz des Gegenübers und eine Gesprächsatmosphäre auf Augenhöhe. Darin unterscheiden sich trans Personen nicht von cis Personen. Allerdings führten die bisherigen Vorgaben zum Ablauf einer Behandlung zuweilen dazu, dass die üblichen Algorithmen der klinischen Arbeit (Anamnese, Diagnostik, Dokumentation, Behandlungsplanung) vernachlässigt wurden. Auch können Übertragungsprozesse im Kontakt mit trans Personen die Verunsicherung und die Ambivalenzen bei den Behandler_innen bedingen bzw. verstärken. Für einen konstruktiv auf die Behandlung bezogenen Umgang mit den eigenen Affekten braucht es allerdings keine Leitlinie, sondern im Bedarfsfall eher Intervision oder Supervision. Schließlich geht es der Leitlinie auch um die Rückbesinnung auf klinisch-therapeutische Expertise, deren Grundlagen und Anspruch sich nicht ändern, wenn eine trans Person zur Behandlung kommt. 
Praxisrelevant wird zudem die Überarbeitung der Begutachtungsrichtlinie des MDS (2009), der dafür das Kompetenzzentrum Psychiatrie und Psychotherapie (KCPP) beauftragt hat. Wie sich die Leitlinie auf die sozialmedizinische Bewertung von Anträgen auf Kostenübernahme transitionsunterstützender Behandlungen auswirken wird, ist bis dato jedoch unklar. Ebenso unklar sind die sozialrechtlichen Auswirkungen. Konkret geht es um die Frage, wie die ICD-11-Diagnose Geschlechtsinkongruenz in das deutsche Gesundheitssystem adaptiert wird. Entscheidend wird sein, ob die neue H-Kategorie „Umstände, die die sexuelle Gesundheit beeinflussen“ (conditions related to sexual health) dem §27 SGB-V Krankenbehandlung zugeordnet wird.

Weitere Rezeptionen der Leitlinie, sowohl von Kolleg_innen, die sie in der klinischen Arbeit praktisch anwenden, als auch von Forschenden, die sich mit der Leitlinie aus einer sozialwissenschaftlichen und/oder gender- sowie queertheoretischen Perspektive auseinandersetzen, werden mit Interesse erwartet: Dazu einladen möchte der Call for Papers (Nieder und Strauß 2019, in diesem Heft).

\section{Interessenkonflikt}

Für die Autoren gilt analog die Erklärung zu den Interessenkonflikten, so wie sie im Zusammenhang mit der S3-Leitlinie auf der entsprechenden AWMF-Seite genannt wird.

\section{Danksagung}

Bei der Leitlinienentwicklung waren viele Menschen tatkräftig und engagiert beteiligt. Ein großer Dank geht an Cathleen Muche-Borowski, die die Durchführung der Evidenzbewertung, die strukturierte Vergabe von Evidenzgraden sowie die Formulierung und Konsentierung der Empfehlungen seitens der AWMF methodisch unterstützt hat. Ebenso danken wir Sarah Barkowski, die uns bei der systematischen Literaturrecherche methodisch geholfen hat, und Andreas Köhler, der die öffentliche Online-Kommentierung des Leitlinienentwurfs bereitgestellt und ausgewertet sowie die Konsensuskonferenzen protokolliert hat. Des Weiteren danken wir allen, die die Möglichkeit zur Online-Kommentierung des Entwurfs der Leitlinie genutzt haben. Ein Dank geht auch an die Bundespsychotherapeutenkammer, die der Leitliniengruppe in Berlin Räumlichkeiten für die Konsensuskonferenzen zur Verfügung gestellt hat. Schließlich gilt ein großer Dank allen Mitgliedern der Leitliniengruppe, die sich mit Engagement und Zeit der Leitlinie verpflichtet haben. Im Einzelnen besonders gedankt sei Michael Berner, Peer Briken, Werner Ettmeier, David Garcia, Dominik Groß, Annette-Kathrin Güldenring, Mari Günther, Josefine Lorenzen, Hagen Löwenberg, Lieselotte Mahler, Wilhelm F. Preuss, Hertha Richter-Appelt, Arn Sauer, Kurt Seikowski, Christian Vogel und Katharina Woellert.

\section{Literatur}

American Psychiatric Association. Diagnostic and Statistical Manual of Mental Disorders. 5th Edition. Washington, DC: APA 2013

American Psychological Association. Guidelines for Psychological Practice with Transgender and Gender Nonconforming People. Am Psychol 2015; 70: 832-864
Ärztliches Zentrum für Qualität in der Medizin. Deutsches Instrument zur methodischen Leitlinien-Bewertung (DELBI). Arbeitsgemeinschaft der Wissenschaftlichen Medizinischen Fachgesellschaften. Düsseldorf, Berlin: AWMF und ÄZQ 2008 [Als Online-Dokument: http://www. leitlinien.de/mdb/edocs/pdf/literatur/delbi-fassung-2005-2006domaene-8-2008.pdf]

AWMF. AWMF-Regelwerk „Leitlinien“. Berlin: AWMF 2012 [Als Online-Dokument://www.awmf.org/leitlinien/awmf-regelwerk.html]

Barker H, Wylie KR. Are the Criteria for the 'Real-Life Experience' (RLE) Stage of Assessment for GID Useful to Patients and Clinicians? Int J Transgend 2008; 10: 121-131

Becker S, Bosinski HAG, Clement U, Eicher W, Goerlich TM, Hartmann U, Kockott G, Langer D, Preuss WF, Schmidt G, Springer A, Wille R. Standards der Behandlung und Begutachtung von Transsexuellen der Deutschen Gesellschaft für Sexualforschung, der Akademie für Sexualmedizin und der Gesellschaft für Sexualwissenschaft. Z Sexualforsch 1997; 10: 147-156

Bockting WO. Psychotherapy and the Real-Life Experience: From Gender Dichotomy to Gender Diversity. Sexologies 2008; 17: 211-224

Bockting WO, Knudson G, Goldberg JM. Counseling and Mental Health Care for Transgender Adults and Loved Ones. Int J Transgend 2007; 9: 35-82

Borras L, Huguelet P, Eytan A. Delusional "Pseudotranssexualism" in Schizophrenia. Psychiatry 2007; 70: 175-179

Byne W, Bradley SJ, Coleman E, Eyler AE, Green R, Menvielle EJ, Meyer-Bahlburg HF, Pleak RR, Tompkins DA. Report of the American Psychiatric Association Task Force on Treatment of Gender Identity Disorder. Arch Sex Behav 2012; 41: 759-796

Campo JM, Nijman H, Evers C, Merckelbach H, Decker I. Gender Identity Disorders as a Secondary Symptom of Psychosis, and in Particular, Schizophrenia. Ned Tijdschr Geneeskd 2001; 145: 1876-1880

Caspari D, Sittinger H, Lang B. Transsexualism and Schizophrenia - Problems in Preparing Expertises for the German Transsexuals Act. Psychiatr Prax 1999; 26: 89-92

Coleman E, Bockting W, Botzer M, Cohen-Kettenis PT, De Cuypere G, Feldman J, Fraser L, Green J, Knudson G, Meyer WJ, Monstrey S, Adler RK, Brown GR, Devor AH, Ehrbar R, Ettner R, Eyler E, Garofalo R, Karasic $\mathrm{DH}$, Lev Al, Mayer G, Meyer-Bahlburg HF, Hall BP, Pfaefflin F, Rachlin K, Robinson B, Schechter LS, Tangpricha V, van Trotsenburg M, Vitale A, Winter S, Whittle S, Wylie KR, Zucker K. Standards of Care for the Health of Transsexual, Transgender, and Gender-Nonconforming People, Version 7. Int J Transgend 2012; 13: 165-232

Commander M], Dean C. Symptomatic Trans-Sexualism. Br ] Psychiatry 1990; 156: 894-896

De Cuypere G, Janes C, Rubens R. Psychosocial Functioning of Transsexuals in Belgium. Acta Psychiatr Scand 1995; 91: 180-184

Deutsch MB. Use of the Informed Consent Model in the Provision of CrossSex Hormone Therapy: A Survey of the Practices of Selected Clinics. Int J Transgend 2012; 13: 140-146

[DGfS] Deutsche Gesellschaft für Sexualforschung. Geschlechtsinkongruenz, Geschlechtsdysphorie und Trans-Gesundheit: S3-Leitlinie zur Diagnostik, Beratung und Behandlung. Version 1.1 (22.02.2019). Berlin: AWMF 2018 [Als Online-Dokument: https://www.awmf.org/leitlinien/ detail/II/138-001.html]

DGSMTW. Stellungnahme zur Leitlinie „Geschlechtsinkongruenz, Geschlechtsdysphorie und Trans-Gesundheit: S3-Leitlinie zur Diagnostik, Beratung und Behandlung (AWMF-Registernr. 138/001)“. Berlin: DGSMTW 2018

Dhejne C, Van Vlerken R, Heylens G, Arcelus J. Mental Health and Gender Dysphoria: A Review of the Literature. Int Rev Psychiat 2016; 28: 44-57

Ellis SJ, McNeil J, Bailey L. Gender, Stage of Transition and Situational Avoidance: A UK Study of Trans People's Experiences. Sex Rel Ther 2014; 29: 351-364

Garcia Nuñez D, Nieder TO. Geschlechtsinkongruenz und -dysphorie: Konzepte und Behandlungsempfehlungen für Trans ${ }^{*}$ Menschen. Gynäkol Endokrinol 2017; 15: 5-13 
Gomez-Gil E, Trilla A, Salamero M, Godas T, Valdes M. Sociodemographic, Clinical, and Psychiatric Characteristics of Transsexuals from Spain. Arch Sex Behav 2009; 38: 378-392

Gomez-Gil E, Zubiaurre-Elorza L, de Antonio IE, Guillamon A, Salamero M. Determinants of Quality of Life in Spanish Transsexuals Attending a Gender Unit before Genital Sex Reassignment Surgery. Qual Life Res 2014; 23: 669-676

Hamm JA, Sauer AT. Perspektivenwechsel: Vorschläge für eine menschenrechts- und bedürfnisorientierte Trans* -Gesundheitsversorgung. Z Sexualforsch 2014; 27: 4-30

Hawkins LA. Gender Identity Development among Transgender Youth: A Qualitative Analysis of Contributing Factors. Chester, PA: Widener University 2009

Hembree WC, Cohen-Kettenis PT, Gooren L, Hannema SE, Meyer WJ, Murad MH, Rosenthal SM, Safer JD, Tangpricha V, T'Sjoen GG. Endocrine Treatment of Gender-Dysphoric/Gender-Incongruent Persons: An Endocrine Society Clinical Practice Guideline. J Clin Endocrinol Metab 2017; 102: 3869-3903

Hepp U, Kraemer B, Schnyder U, Miller N, Delsignore A. Psychiatric Comorbidity in Gender Identity Disorder. J Psychosom Res 2005; 58: 259-261

Heylens G, Elaut E, Kreukels BPC, Paap MCS, Cerwenka S, Richter-Appelt H, Cohen-Kettenis PT, Haraldsen IR, De Cuypere G. Psychiatric Characteristics in Transsexual Individuals: Multicentre Study in Four European Countries. Br J Psychiatry 2014; 204: 151-156

Hrobjartsson A, Boutron I, Turner L, Altman DG, Moher D. Assessing Risk of Bias in Randomised Clinical Trials Included in Cochrane Reviews: The Why Is Easy, the How Is a Challenge. Cochrane Database Syst Rev 2013; doi: 10.1002/14651858.ed000058

jiloha RC, Bathla JC, Bawbja A, Gupta V. Trans-Sexualism in Schizophrenia : A Case Report. Indian J Psychiatry 1998; 40: 186-188

Kenagy GP, Hsieh CM. Gender Differences in Social Service Needs of Transgender People. J Soc Serv Res 2005; 31: 1-21

Klein V, Brunner F, Nieder TO, Reed G, Briken P. Diagnoseleitlinien sexueller Störungen in der International Classification of Diseases and Related Health Problems (ICD)-11: Dokumentation des Revisionsprozesses. Z Sexualforsch 2015; 28: 363-373

Koehler A, Eyssel J, Nieder TO. Genders and Individual Treatment Progress in (Non-)Binary Trans Individuals. J Sex Med 2018; 15: 102-113

Köhler A, Becker I, Richter-Appelt H, Cerwenka S, Kreukels B, van de Grift T, Elaut E, Heylens G, Nieder TO. Behandlungserfahrungen und soziale Unterstützung bei Personen mit Geschlechtsinkongruenz/Geschlechtsdysphorie: Eine ENIGI 5-Jahres-Follow-Up-Studie in drei europäischen Ländern. Psychother Psych Med 2019; doi: 10.1055/a-0806-6892

Kuper LE, Nussbaum R, Mustanski B. Exploring the Diversity of Gender and Sexual Orientation Identities in an Online Sample of Transgender Individuals. J Sex Res 2012; 49: 244-254

Laszig P, Knauss W, Clement U. Psychotherapeutische Begleitung einer transsexuellen Entwicklung. Z Sexualforsch 1995; 8: 24-38

Levine SB, Solomon A. Meanings and Political Implications of Psychopathology in a Gender Identity Clinic: A Report of 10 Cases. J Sex Marital Ther 2009; 35: 40-57

Loos F, Köhler A, Eyssel J, Nieder TO. Subjektive Indikatoren des Behandlungserfolges und Diskriminierungserfahrungen in der trans* Gesundheitsversorgung. Qualitative Ergebnisse einer Online-Befragung. Z Sexualforsch 2016; 29: 205-223

Löwenberg H, Ettmeier W. Die Rolle der Psychotherapie in einem integrativen Behandlungsansatz bei Geschlechtsdysphorie. Z Sexualforsch 2014; 27: 44-58

[MDS] Medizinischer Dienst des Spitzenverbandes Bund der Krankenkassen. Grundlagen der Begutachtung. Begutachtungsanleitung. Geschlechtsangleichende Maßnahmen bei Transsexualität. Essen: MDS 2009

Monro S. Theorizing Transgender Diversity: Towards a Social Model of Health. Sex Relation Ther 2000; 15: 33-45

Murad MH, Elamin MB, Garcia MZ, Mullan RJ, Murad A, Erwin PJ, Montori VM. Hormonal Therapy and Sex Reassignment: A Systematic Review and Meta-Analysis of Quality of Life and Psychosocial Outcomes. Clin Endocrinol 2010; 72: 214-231

Nieder TO, Briken P, Güldenring AK. Geschlechtsinkongruenz, -dysphorie und Trans*-Gesundheit: Eine aktuelle Übersicht zur Diagnostik und Behandlung. InFo Neurol Psychiat 2016; 18: 37-45

Nieder TO, Briken P, Richter-Appelt $\mathrm{H}$. Transgender, Transsexualität und Geschlechtsdysphorie: Aktuelle Entwicklungen in Diagnostik und Therapie. Psychother Psych Med 2014a; 64: 232-245

Nieder TO, Cerwenka S, Richter-Appelt H. Nationale und internationale Ansätze der Diagnostik und Versorgung von Menschen mit Transsexualität oder Geschlechtsdysphorie. In: Richter-Appelt H, Nieder TO, Hrsg. Transgender-Gesundheitsversorgung. Eine kommentierte Herausgabe der Standards of Care der World Professional Association for Transgender Health. Gießen: Psychosozial 2014b; 19-43

Nieder TO, Strauß B. Leitlinie zur Diagnostik, Beratung und Behandlung bei Geschlechtsdysphorie. Stand der aktuellen Entwicklungen.

Z Sexualforsch 2014; 27: 59-76

Nieder TO, Strauss B. Transgender Health Care in Germany: Participatory Approaches and the Development of a Guideline. Int Rev Psychiat 2015; 27: 416-426

Nieder TO, Strauß B. Call for Papers. Geschlechtsinkongruenz, Geschlechtsdysphorie und Trans-Gesundheit: Beiträge zur S3-Leitlinie. Z Sexualforsch 2019; 32: 69

Nuttbrock L, Bockting W, Rosenblum A, Hwahng S, Mason M, Macri M, Becker J. Gender Abuse, Depressive Symptoms, and HIV and Other Sexually Transmitted Infections among Male-to-Female Transgender Persons: A Three-Year Prospective Study. Am J Public Health 2013; 103: $300-307$

Poteat T, German D, Kerrigan D. Managing Uncertainty: A Grounded Theory of Stigma in Transgender Health Care Encounters. Soc Sci Med 2013; 84: 22-29

Radix A, Eisfeld J. Informierte Zustimmung in der Trans*-Gesundheitsversorgung. Z Sexualforsch 2014; 27: 31-43

Reisner SL, Poteat T, Keatley J, Cabral M, Mothopeng T, Dunham E, Holland CE, Max R, Baral SD. Global Health Burden and Needs of Transgender Populations: A Review. Lancet 2016; 388: 412-436

Richards C, Barker M, Lenihan P, lantaffi A. Who Watches the Watchmen? A Critical Perspective on the Theorization of Trans People and Clinicians. Feminism Psychol 2014; 24: 248-258

Richter-Appelt H, Nieder TO. Transgender-Gesundheitsversorgung. Eine kommentierte Herausgabe der Standards of Care der World Professional Association for Transgender Health. Gießen: Psychosozial 2014

Schulz SL. Gender Identity: Pending? Identity Development and Health Care Experiences of Transmasculine/Genderqueer Identified Individuals. Berkeley, CA: University of California, Berkeley 2012

Sutcliffe PA, Dixon S, Akehurst RL, Wilkinson A, Shippam A, White S, Richards R, Caddy CM. Evaluation of Surgical Procedures for Sex Reassignment: A Systematic Review. J Plast Reconstr Aesthet Surg 2009; 62: 294-306

World Health Organisation. International Classification of Diseases 11th Revision. Genf: WHO 2018

World Medical Association. WMA Statement on Transgender People. FernayVoltaire: WMA 2015

Xavier JM, Bobbin M, Singer B, Budd E. Needs Assessment of Transgendered People of Color Living in Washington, DC. Int J Transgend 2005; 8: 31-47

Yerke AF, Mitchell V. Am I Man Enough Yet? A Comparison of the Body Transition, Self-Labeling, and Sexual Orientation of Two Cohorts of Femaleto-Male Transsexuals. Int J Transgend 2011; 13: 64-76

Zucker KJ, Cohen-Kettenis PT, Drescher ], Meyer-Bahlburg HF, Pfäfflin F, Womack WM. Memo Outlining Evidence for Change for Gender Identity Disorder in the DSM-5. Arch Sex Behav 2013; 42: 901-914 